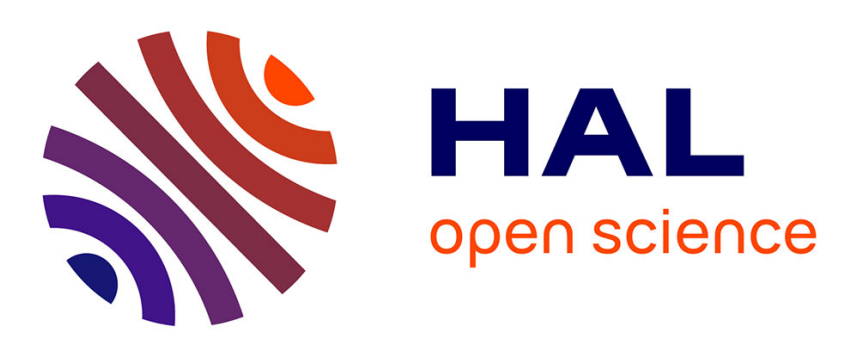

\title{
Réceptivité des sols aux fusarioses vasculaires: méthode statistique d'analyse des résultats
}

Antoine Corman, Yvonne Couteaudier, Michèle Zegerman, Claude

Alabouvette

\section{- To cite this version:}

Antoine Corman, Yvonne Couteaudier, Michèle Zegerman, Claude Alabouvette. Réceptivité des sols aux fusarioses vasculaires: méthode statistique d'analyse des résultats. Agronomie, 1986, 6 (8), pp.751-757. hal-00884933

\section{HAL Id: hal-00884933 https://hal.science/hal-00884933}

Submitted on 1 Jan 1986

HAL is a multi-disciplinary open access archive for the deposit and dissemination of scientific research documents, whether they are published or not. The documents may come from teaching and research institutions in France or abroad, or from public or private research centers.
L'archive ouverte pluridisciplinaire HAL, est destinée au dépôt et à la diffusion de documents scientifiques de niveau recherche, publiés ou non, émanant des établissements d'enseignement et de recherche français ou étrangers, des laboratoires publics ou privés. 


\title{
Réceptivité des sols aux fusarioses vasculaires : méthode statistique d'analyse des résultats
}

\author{
Antoine CORMAN, Yvonne COUTEAUDIER (*), Michèle ZEGERMAN \& Claude ALABOUVETTE (*) $\left({ }^{\mathrm{l}}\right)$ \\ Laboratoire de Biométrie L.A. 243, Université Claude Bernard, Lyon 1, 43, bd du II Novembre 1918, F 69622 \\ Villeurbanne Cedex \\ (*) I.N.R.A., Station de Recherches sur la Flore pathogène dans le Sol, 17, rue Sully, F 21034 Dijon Cedex
}

RÉSUMÉ

\begin{abstract}
Un test biologique permettant de comparer la réceptivité de différents sols aux fusarioses vasculaires a été décrit précédemment (AlABOUvETTE et al., 1982). Dans cet article, nous proposons un traitement statistique des données permettant un classement des sols et leur comparaison sur des bases quantitatives. La probabilité de survie des plantes a été modélisée à partir des résultats d'une expérience dans laquelle différentes souches de Fusarium non pathogènes ont été comparées pour leur aptitude à établir la résistance dans des sols traités à la chaleur. Ce modèle est une extension à plusieurs variables explicatives du modèle Logit et prend en compte 2 facteurs quantitatifs, la concentration initiale d'inoculum et le temps, et 1 facteur qualitatif, l'effet du traitement. Dans tous les cas, la variabilité des données est supérieure à la variabilité déduite du modèle binomial, mais les écarts entre probabilité de survie théorique et probabilité de survie observée sont sans importance pratique pour ce type d'essai biologique. La validité du modèle a ensuite été testée sur les données d'une expérimentation relative aux mécanismes de la résistance des sols de Châteaurenard.

Il ressort de cette étude que la probabilité de survie des plantes n'est pas constante au cours du temps. Ce modèle constitue un outil méthodologique indispensable à l'analyse des données relatives à l'intensité de la compétition nutritive dans les sols et à la sélection des différents microorganismes antagonistes utilisables dans la lutte contre les fusarioses vasculaires.
\end{abstract}

Mots clés additionnels : Modélisation, probabilité de survie, Fusarium, résistance, lutte biologique.

A biological test allowing comparison of the receptivity of different soils to fusarium wilts has been described previously (AlabouvetTE et al., 1982). This paper presents a statistical method for analyzing such data, enhancing the comparison and classification of the soils on a quantitative basis. The probability of plant survival has been modeled using data from an experiment in which different strains of non pathogenic Fusarium were compared for their ability to establish suppressiveness in different heat-treated soils. This model takes into account initial inoculum and time as quantitative factors and treatment as a qualitative one and is a multivariate extension of the classical Logit transformation. In all cases, the variability of the data was greater than that deduced from the binomial model, but differences between experimental and calculated survival probabilities were always without practical significance. The model was then fitted to data from a study on the mechanisms of suppressiveness of the Chateaurenard soil. An important feature of the different fits is that survival probability varied significantly during the time course of the experiment. The model represents a useful tool for comparing different intensities of competition in soils and for screening efficient strains for biological control of fusarium wilts.

Additional key words : Statistical modelling, survival probability, Fusarium, suppressiveness, biological control.

\section{INTRODUCTION}

Dans un article précédent (ALABOUVETTE et al., 1982), nous avons proposé un test biologique permettant de comparer la réceptivité de différents sols aux

(1) Les tirés à part doivent être demandés à Dijon. fusarioses vasculaires. Cette méthode consiste à introduire des doses croissantes d'un inoculum de Fusarium oxysporum f. sp. lini dans les sols à comparer, puis à cultiver la plante-hôte sensible dans des conditions contrôlées favorables à l'expression de la maladie. Les observations effectuées au cours de la culture permettent d'établir une courbe de mortalité à une date donnée en fonction des doses croissantes d'inoculum. La comparaison graphique des différentes cour- 
bes obtenues par rapport à un sol sensible ou un sol résistant de référence permet d'apprécier la réceptivité des sols étudiés, mais le classement précis des sols et leur comparaison sur des bases objectives nécessite un traitement statistique des données. Il était donc nécessaire d'élaborer un modèle mathématique applicable à ce protocole d'étude à partir de différentes hypothèses et en tenant compte de la nature des données expérimentales.

La mise au point du modèle a été réalisée à partir d'une expérimentation au cours de laquelle était comparée l'aptitude de différentes souches de Fusarium non pathogènes à diminuer la réceptivité d'un sol traité à la chaleur. Sa validité a ensuite été testée sur les résultats obtenus dans une expérimentation portant sur l'étude des mécanismes de la résistance du sol de Châteaurenard.

\section{MATÉRIEL ET MÉTHODES}

\section{A. Matériel biologique et méthodes expérimentales}

\section{Aptitude des souches à établir la résistance dans un sol désinfecté}

Trois expérimentations ont été réalisées simultanément dans 3 sols différents en confrontant un $F$. oxysporum f. sp. lini à différentes souches de $F$. oxysporum et $F$. solani. Ces souches issues d'un repiquage monospore sont les suivantes :
- F. o. f. sp. lycopersici (Fol 8) : agent de la fusariose vasculaire de la tomate.

- Fo 20 et Fo $47: F$. oxysporum non pathogènes isolés du sol de Châteaurenard.

- Fs 2, Fs 3, Fs 52 et Fs $59: F$. solani non pathogènes isolés du sol de Châteaurenard.

Les 3 sols utilisés diffèrent par leurs caractéristiques physico-chimiques, agronomiques et culturales (tabl. 1).

Chaque souche est incorporée sous forme d'inoculum-talc (TELLO-MARQUINA \& ALABOUVETTE, 1984) au sol traité à la chaleur $\left(1 \mathrm{~h}, 100^{\circ} \mathrm{C}\right)$. Après $15 \mathrm{j}$ d'incubation, les mélanges sont infestés avec un inoculum de $F$. o. f. sp. lini aux doses de 800, 1600 , 3200 et 6400 propagules/g de sol ; chaque traitement est réparti en 6 pots de $250 \mathrm{ml}$ dans lesquels sont semées 10 graines de lin d'une variété sensible à la maladie, «Hera ». Les expérimentations sont conduites en chambre climatisée pendant 8 à 9 semaines en conditions parfaitement contrôlées (ALABOUVETTE et al., 1982). Une notation du nombre de plantes survivantes est effectuée chaque semaine.

\section{Analyse des mécanismes de la résistance du sol de Châteaurenard}

Différents traitements sont appliqués au sol de Châteaurenard afin d'y rétablir la résistance, après sa suppression par traitement à la chaleur $\left(1 \mathrm{~h}, 100^{\circ} \mathrm{C}\right)$. On introduit dans le sol traité soit une souche de $F$. oxys-

TABLEAU 1

Caractéristiques physico-chimiques des sols de Châteaurenard, Dijon et Ouroux.

Physico-chemical characteristics of soils from Châteaurenard, Dijon and Ouroux.

\begin{tabular}{|c|c|c|c|c|c|}
\hline Granulométrie & & & Châteaurenard & Dijon & Ouroux \\
\hline Sables grossiers $(2 \mathrm{~mm}-0,2 \mathrm{~mm})$ & & p. 1000 & 5 & 65 & 787 \\
\hline Sables fins $\quad(0,2 \mathrm{~mm}-0,05 \mathrm{~mm})$ & & p. 1000 & 98 & 49 & 96 \\
\hline Limon grossier $(0,06 \mathrm{~mm}-0,02 \mathrm{~mm})$ & & p. 1000 & 192 & 79 & 19 \\
\hline Limon $\quad(0,02 \mathrm{~mm}-0,002 \mathrm{~mm})$ & & p. 1000 & 152 & 254 & 23 \\
\hline Argile $\quad(<0,002 \mathrm{~mm})$ & & p. 1000 & 227 & 351 & 72 \\
\hline $\mathrm{pH}$ eau & & & 7,9 & 7,9 & 7,6 \\
\hline Carbone organique (méthode Anne) & & p. 1000 & 22,6 & 14,7 & 4,8 \\
\hline Matières organiques & & p. 1000 & 38,8 & 25,2 & 8,2 \\
\hline Azote Kjeldahl & & p. 1000 & 2,93 & 1,58 & 0,52 \\
\hline $\mathrm{C} / \mathrm{N}$ & & & 7,71 & 9,30 & 9,23 \\
\hline Calcaire total & & p. 1000 & 318 & 38 & 3 \\
\hline Calcaires actifs & & p. 1000 & 92 & - & 79 \\
\hline Acide phosphorique & & p. 1000 & 0,29 & 0,23 & 0,02 \\
\hline Capacité d'échange & M eq. & p. 100 & 12,7 & 18,2 & 3,9 \\
\hline Calcium échangeable & M eq. & p. 100 & 37,4 & 38,5 & 6,0 \\
\hline Magnésium échangeable & M eq. & p. 100 & 1,96 & 1,09 & 0,24 \\
\hline Potassium échangeable & $\mathrm{M}$ eq. & p. 100 & 0,915 & 0,624 & 0,085 \\
\hline Manganèse échangeable & & p.p.m. & 3,3 & 3,7 & 0,8 \\
\hline Aluminium échangeable & $\mathrm{M}$ eq. & p. 100 & 0,2 & 0,3 & 0,1 \\
\hline Fe soluble & & p. 100 & 0,76 & 1,53 & 0,61 \\
\hline Calcium total & & p. 100 & 13,13 & 2,16 & 0,25 \\
\hline Magnésium total & & p. 100 & 0,80 & 0,46 & 0,14 \\
\hline Potassium total & & p. 100 & 0,96 & 1,30 & 2,56 \\
\hline Sodium total & & p. 100 & 0,65 & 0,43 & 0,55 \\
\hline Cuivre total & & p.p.m. & 43,5 & 24,1 & 5,9 \\
\hline Fe total & & p. 100 & 2,19 & 2,99 & 0,99 \\
\hline Manganèse total & & p.p.m. & 623 & 761 & 173 \\
\hline Aluminium total & & p. 100 & 4,59 & 5,50 & 3,63 \\
\hline Cuivre extractible E.D.T.A. & & p.p.m. & 14,2 & 4,0 & 1,0 \\
\hline Manganèse extractible E.D.T.A. & & p.p.m. & 13,3 & 16,6 & 8,5 \\
\hline Zinc extractible E.D.T.A. & & p.p.m. & 2,4 & 6,3 & 1,5 \\
\hline
\end{tabular}


porum non pathogène (Fo 47 à la dose de $2.10^{4}$ propagules/g de sol) soit une mycoflore complexe (COUTEAUDIER et al., 1985) exempte de Fusarium. Un $3^{\mathrm{e}}$ traitement consiste à introduire la souche de $F$, oxysporum non pathogène dans le sol autoclavé déjà colonisé par la mycoflore complexe.

Après 3 semaines d'incubation, le pathogène $F$. $o$. f. sp. lini est introduit aux doses de 500, 1000,2000 , 4000 et 8000 propagules/g de sol, puis l'expérimentation est conduite dans les mêmes conditions que précédemment.

\section{B. Analyses statistiques}

La maladie ne se manifestant qu'à partir de la $3^{\mathrm{e}}$ semaine de culture, le temps $\mathrm{t}=3$ semaines est considéré comme le $t_{0}$ dans les calculs. Ceux-ci sont effectués sur la base de 60 plantes par traitement, les 6 pots de 10 plantes considérés a priori comme homogènes ayant été regroupés afin d'éviter les inconvénients liés aux petits effectifs. Les données ont été traitées par le logiciel GLIM (Generalised Linear Interactive Modelling, distribué par le Numerical Algorithm Group, Oxford, Grande-Bretagne) implanté sur l'Eclipse S 140 Data General du Laboratoire de Biométrie de l'Université Claude Bernard à Lyon. (Les auteurs tiennent à la disposition des lecteurs intéressés l'ensemble des données expérimentales ainsi que les calculs et graphiques obtenus à l'aide du logiciel GLIM).

Il existe a priori 3 sources de variations : 2 quantitatives, la dose d'inoculum et le temps de culture, et 1 qualitative, l'effet du traitement (dans la $1^{\text {re }}$ expérimentation l'effet souche non pathogène). D'autre part, les notations du nombre de plantes vivantes étant faites sous forme cumulative au cours du temps, les données successives peuvent être rendues indépendantes entre elles par le calcul, pour chaque instant $t$, de la quantité :

$$
\hat{\mathbf{P}}_{\mathrm{t}}=\frac{\text { Nombre de plantes vivantes à } \mathrm{t}\left(\mathrm{N}_{\mathrm{t}}\right)}{\text { Nombre de plantes vivantes à } \mathrm{t}-1\left(\mathrm{~N}_{\mathrm{t}-1}\right)}
$$

$\hat{\mathrm{P}}_{\mathrm{t}}$ est un estimateur de $\mathrm{P}_{\mathrm{t}}$, probabilité qu'une plante soit vivante en $t$, sachant qu'elle l'est en $t-1$, pour un traitement et une dose d'inoculum donnés. Cette quantité $\mathbf{P}$ est modélisée par l'équation :

$$
\log \frac{P}{1-P}=f(D, T, S)
$$

où $f$ représente une fonction linéaire en ses paramètres, $\mathrm{D}$ est la densité initiale de l'inoculum pathogène, $\mathrm{T}$ est le temps (compté à partir de la $3^{\mathrm{e}}$ semaine), $\mathrm{S}$ l'effet-souche. (1) est un modèle Logit à plusieurs variables explicatives, dérivé du modèle classique :

$$
\log \frac{P}{1-P}=\alpha D+\beta(\alpha \text { et } \beta \text { : paramètres })
$$

L'estimation des paramètres du modèle par la méthode du maximum de vraisemblance s'obtient par une méthode itérative compte tenu du fait que $\mathrm{N}_{1}$ suit une loi binominale $\left(\mathrm{N}_{\mathrm{t}-\mathrm{l}}, \mathrm{P}_{\mathrm{t}}\right)$. A l'issue de l'estimation, le programme fournit les valeurs des différents paramètres, leur écart-type, la matrice des corrélations et la valeur des résidus. La valeur de l'ajustement est mesurée par la déviance qui est une quantité égale à moins 2 fois le $\log$ du rapport de vraisemblance et qui est distribuée asymptotiquement selon une loi de $\chi^{2}$.

En raison du nombre élevé de degrés de liberté (115 au minimum), cette approximation est justifiée ainsi que la réalisation de tests de STUDENT pour juger de la pertinence d'un des paramètres du modèle (BAKER \& NELDER, 1978).

L'équation (1) peut s'écrire, de façon plus détaillée, pour le traitement $\mathrm{i}$ :

$$
\log \frac{P}{1-P}=a+b_{i}+g(D)+h(T)
$$

avec a, paramètre d'《 ordonnée à l'origine 》générale ; $b_{i}$, paramètre représentant l'effet du traitement $i$ $(i \geqslant 2)$ sur le témoin (l'effet du traitement 1 étant fixé à 0 , celui-ci sera toujours le témoin désinfecté); g et $h$, fonctions de $D$ et $T$ respectivement, linéaires en leurs paramètres.

Il est possible de tester la signification d'une variable en analysant l'effet de son introduction dans le modèle sur la valeur de la déviance (par un test $\chi^{2}$, théoriquement valable asymptotiquement). Toujours en s'appuyant sur les propriétés asymptotiques, la comparaison entre les $b_{i}$ (effet traitement) est réalisée par le calcul de la quantité :

$$
\frac{\left|\hat{b}_{i}-\hat{b}_{j}\right|}{\sqrt{\operatorname{var}\left(\hat{b}_{i}-\hat{b}_{j}\right)}} \quad(i, j \geqslant 2)
$$

où var $\left(\hat{b}_{\mathrm{i}}-\hat{\mathrm{b}}_{\mathrm{j}}\right)$ est calculée à partir de la matrice de covariances.

Cette quantité est comparée à un seuil $\mathrm{Z}$, quantile de la loi de Gauss, correspondant à une probabilité $\frac{2 \alpha}{n(n-1)}$ d'être dépassée en valeur absolue (test bilatéral) si $\mathrm{H}_{0}$ est vraie, pour un risque global $\alpha$ (règle de BONFERONNI avec $\mathbf{n}=$ nombre de traitements).

Le nombre de comparaisons à réaliser est en effet a priori égal à $\frac{\mathrm{n}(\mathrm{n}-1)}{2}$, puisqu'on veut non seulement détecter les traitements qui représentent une amélioration par rapport au témoin, mais également, parmi ces derniers, le (ou les) plus performant(s). On augmente ainsi sans doute exagérément le risque de $2^{\mathrm{e}}$ espèce, mais il est préférable de ne conclure à l'efficacité d'un traitement qu'avec un risque d'erreur très faible, surtout si cela doit déboucher sur des utilisations en lutte biologique.

\section{RÉSULTATS}

\section{A. Effet souche}

Une étude détaillée sera présentée dans le cas du sol de Dijon. Pour les 2 autres sols et pour l'étude des mécanismes de la résistance du sol de Châteaurenard, seuls les résultats finaux seront donnés. 

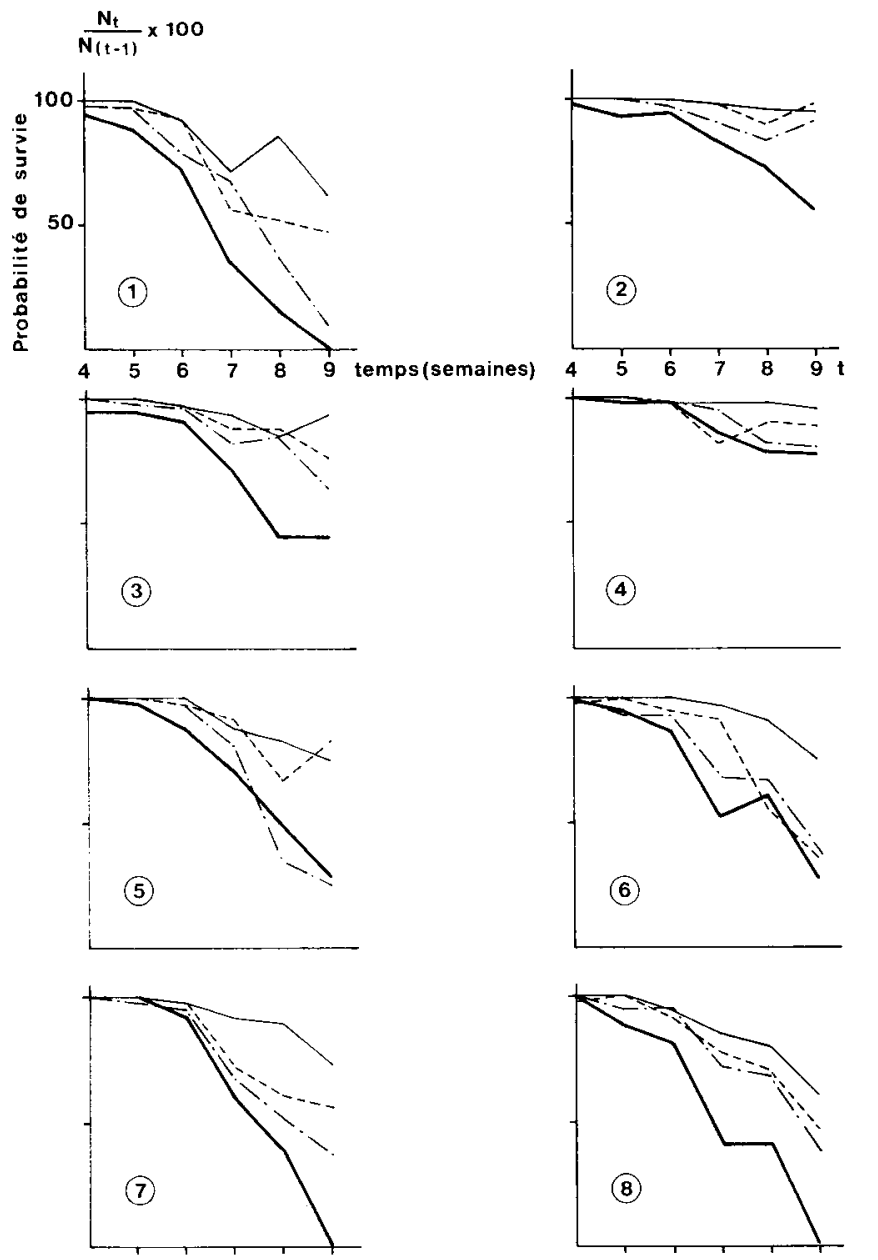

Figure 1

Probabilité de survie des plantes enregistrée au cours du temps en sol de Dijon traité à la chaleur, amendé avec différentes souches de Fusarium non pathogènes $(I:$ sol désinfecté : $2:$ Fol $18 ; 3:$ Fo 20 : $4:$ Fo $47 ; 5:$ Fs $2 ; 6:$ Fs $3 ; 7:$ Fs $52 ; 8:$ Fs 59) puis infesté à 4 doses de F. oxysporum $f$. sp. lini (—_ : $800 ;----\cdots . .$. :

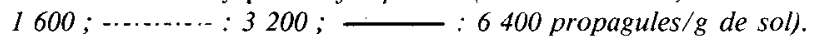

Survival probability against time in heat-treated Dijon soil, amended with different strains of non-pathogenic Fusarium $(1:$ control, 2 : Fol 18. 3: Fo 20, 4 : Fo $47 ; 5:$ Fs 2 ; 6: Fs 3; 7 : Fs 52;8: Fs 59) then infested with $\mathrm{F}$. oxysporum $f$. sp. lini (- 800 ;

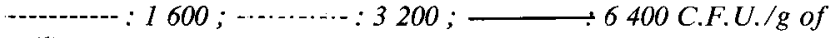
soil).

\section{Sol de Dijon}

La représentation graphique de la probabilité de survie observée pour chaque souche en fonction du temps (fig. 1) met en évidence :

- un très fort effet non linéaire du temps sur cette variable : quelle que soit la souche, la survie diminue lentement jusqu'à la $6^{\mathrm{e}}$ semaine, puis plus rapidement pour les semaines 6 et 7 et se stabilise aux $8^{\text {e }}$ et $9^{\mathrm{e}}$ semaines :

- un effet souche assez net, les $F$. oxysporum (Fol 8 et Fo 47) se différenciant nettement des F. solani ;

- un effet dose dont la variation est irrégulière. On note certains chevauchements des courbes correspondant à 2 doses d'inoculum successives.

Les $1^{\text {ers }}$ essais d'ajustement de l'équation (2) aux données de la figure 1 ont montré que :
- pour la dose, l'utilisation de la fonction $g(D)=\log (D)$ donne les meilleurs résultats. Ceci est conforme aux données de la littérature (BAKER et al., 1967) ;

- la modélisation de l'effet temps est beaucoup plus complexe. La technique suivante a été utilisée : introduction pas à pas dans la fonction $\mathrm{h}(\mathrm{T})$ des puissances successives de $T$ jusqu'à un terme d'exposant 4 , ainsi que de la fonction $\exp (\mathrm{T})$, en testant pour chaque nouvelle introduction son effet sur la valeur de la déviance. Le calcul des écarts-types des paramètres a permis d'éliminer les paramètres dont la valeur était inférieure à leur écart-type, en vérifiant que cette suppression était sans effet sur la valeur de la déviance. Les termes d'exposants 1 et 2 ont ainsi été retirés du modèle.

En définitive, on obtient :

$$
\begin{aligned}
& \mathrm{g}(\mathrm{D})=-0,99 \log (\mathrm{D}) \\
& \mathrm{h}(\mathrm{T})=-0,18 \mathrm{~T}^{3}+0,04 \mathrm{~T}^{4}-0,03 \exp (\mathrm{T}) .
\end{aligned}
$$

L'ensemble des paramètres du modèle figure au tableau 2. Le critère d'ajustement est très réduit par rapport à sa valeur initiale mais le test $\chi^{2}$ reste très significatif (tabl. 2). Cependant l'examen des résidus, que ce soit en fonction de la dose, de la souche ou du temps, ne montre aucune tendance particulière ; leur dispersion est à peu près constante et leur moyenne proche de 0. D'autre part, les valeurs calculées et observées du nombre de plantes vivantes sont en règle générale très proches (fig. 2).

Dans le cas de grands échantillons, la variable $\chi^{2}$ peut prendre des valeurs très supérieures à son espérance car le moindre écart au modèle peut être détecté même si celui-ci est sans importance pratique (BAKER \& NELDER, 1978). D'autre part, la contrainte sur le $\chi^{2}$ (effectifs théoriques $\geqslant 5$ ) n'est pas vérifiée, en particulier lorsque les nombres de survivants ou de morts sont très faibles.

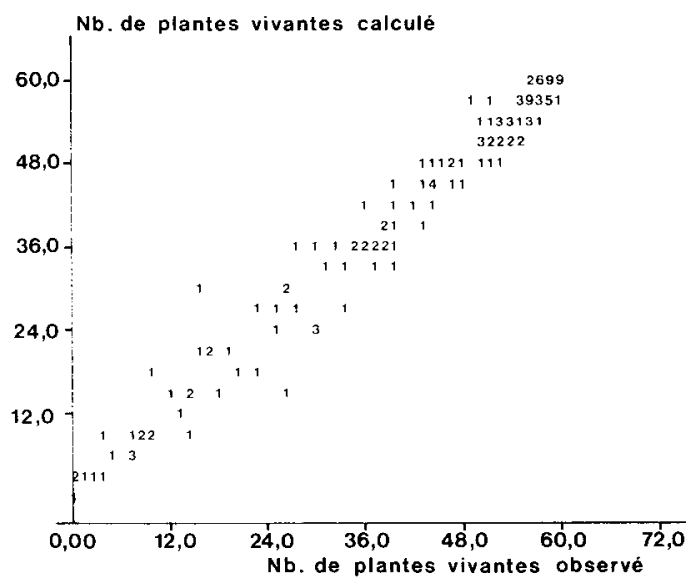

Figure 2

Comparaison du nombre de plantes saines observé et du nombre de plantes saines calculé (192 données) à partir de l'équation

$$
\log \frac{P}{I-P}=a+b_{i}+g(D)+h(T) .
$$

Les chiffres indiquent le nombre de points superposés (9 signifie $\geqslant 9$ ). Comparison between the observed number of healthy plants and the number calculated $(n=192)$ by the equation.

$$
\log \frac{P}{1-P}=a+b_{i}+g(D)+h(T) .
$$

Figures indicate the number of superposed points (9 means $\geqslant 9$ ). 
TABLEAU 2

Valeurs des paramètres (et écart-type) du modèle défini par l'équation $\log \frac{P}{l-P}=a+b_{i}+g(D)+h(T)$ pour les sols de Dijon. Ouroux et Châteaurenard. L'approximation normale $d u \chi^{2}$ a été calculée par

$$
\text { A. } N=\sqrt{2 \overline{\chi^{2}}}-\sqrt{2 d d l-l} .
$$

Parameter values (and standard deviation) of the model defined by the equation $\log \frac{P}{I-P}=a+b_{i}+g(D)+g(T)$ for the soils of Dijon, Ouroux and Châteaurenard. $\chi^{2}$ normal approximution calculated

$$
\text { by } N \cdot A=\sqrt{2} \chi^{2}-\sqrt{2 d d t-1} .
$$

\begin{tabular}{|c|c|c|c|}
\hline \multirow[b]{2}{*}{ Paramètres } & \multirow[b]{2}{*}{ Dijon } & Valeur des paramètres (écart-type) & \multirow[b]{2}{*}{ Châteaurenard } \\
\hline & & Ouroux & \\
\hline$a_{1}$ (témoin) & $12,14(0,55)$ & $8,89(0,57)$ & $11,13(0,64)$ \\
\hline$b_{2}($ Fol 8$)$ & $2,40(0,19)$ & $1,60(0,19)$ & $2,52(0,62)$ \\
\hline$b_{3}(F o 20)$ & $1,54(0,17)$ & $1,93(0,19)$ & $1,88(0,20)$ \\
\hline $\mathrm{b}_{4}(\mathrm{Fo} 47)$ & $2,42(0,19)$ & $2,33(0,20)$ & $2,20(0,21)$ \\
\hline $\mathrm{b}_{5}$ (Fs 2) & $1,09(0,16)$ & $1,65(0,19)$ & $1,94(0,20)$ \\
\hline$b_{6}$ (Fs 3) & $0,87(0,16)$ & $2,41(0,20)$ & $2,0 \quad(0,20)$ \\
\hline $\mathrm{b}_{7}$ (Fs 52) & $0,79(0,16)$ & $1,92(0,19)$ & $1,08(0,18)$ \\
\hline $\mathrm{b}_{8}$ (Fs 59) & $0,57(0,16)$ & $2,53(0,21)$ & $0,98(0,19)$ \\
\hline $\log (\mathrm{D})$ & $-0,99(0,06)$ & $-0,67(0,07)$ & $-0,84(0,07)$ \\
\hline $\mathrm{T}^{3}$ & $-0,18(0,02)$ & $-0,33(0,22)$ & $-0,31(0,02)$ \\
\hline $\mathrm{T}^{4}$ & $0,04(0,005)$ & $0,15(0,01)$ & $0,08(0,006)$ \\
\hline$e^{T}$ & $-0,03(0,006)$ & $-0,39(0,03)$ & $-0,11(0,009)$ \\
\hline $\mathrm{ddl}$ & 180 & 180 & 180 \\
\hline$\chi^{2}\left(\chi^{2}\right.$ modèle minimal $)$ & $271(2388)$ & $341(1936)$ & $347(1810)$ \\
\hline Approximation normale & 4,2 & 7,1 & 7,4 \\
\hline Facteur d'hétérogénéité & 1,5 & 1,9 & 1,92 \\
\hline
\end{tabular}

Enfin, la régression pondérée est réalisée avec l'hypothèse d'une variabilité des données entièrement déterminée par leur nature binomiale. Ceci est une contrainte assez forte car à cette variabilité théorique peut s'en ajouter une autre, d'ordre expérimental. Ainsi FINNEY (1978) propose d'utiliser une variance proportionnelle (plutôt que strictement égale) à la variance binomiale. Le facteur de proportionnalité, appelé facteur d'hétérogénéité, peut être estimé à partir des données (comme dans le cas d'une Loi Normale) par :

$$
\mathrm{S}^{2}=\frac{\chi^{2}}{\text { nombre de ddl }}
$$

Pour ce sol, on obtient $\mathrm{S}^{2}=1,5$. L'utilisation d'un tel facteur introduit évidemment un élément d'incertitude supplémentaire dans les tests réalisés à partir du modèle, bien que l'on puisse le rattacher à la notion de quasi-vraisemblance (BAKER \& NELDER, 1978).

Cependant le modèle défini par l'équation (2) peut être considéré comme satisfaisant si l'on se réfère à l'examen des résidus et de la figure 2, ainsi qu'à la faiblesse de la correction réalisée; les écarts-types ne sont en effet multipliés que par un facteur de 1,22. Les valeurs ainsi obtenues sont reportées dans le tableau 2.

Les souches saprophytes peuvent donc être classées par ordre d'efficacité croissante (tabl. 2) et la signification des différences entre souches (ou entre souches et témoin) peut être testée. Le nombre total de tests est ici a priori de 28 d'où pour $Z$ une valeur de 3,125 . On ne présentera pas la matrice des variancescovariances, mais on peut indiquer que la corrélation entre les $b_{i}$ est pratiquement constante, quel que soit le couple considéré, et de l'ordre de 0,4-0,5 (ceci est d'ailleurs vrai pour les 3 sols). Le calcul exact conduit aux résultats de la figure 3 . On constate que toutes les

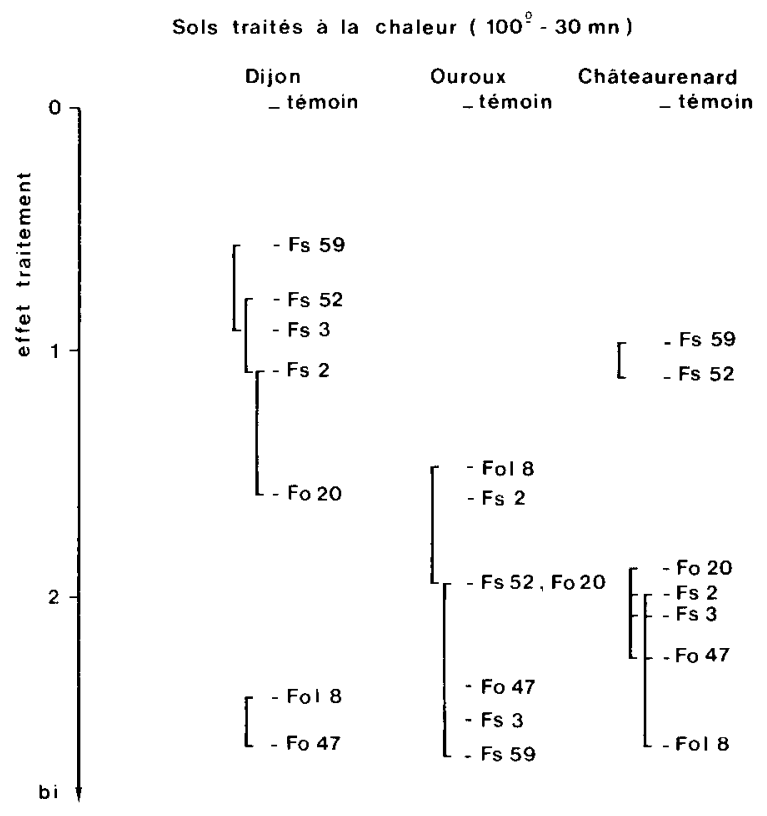

Figure 3

Effet souche en fonction de la valeur prise par le paramètre $b_{i}$ et test de comparaison des $b_{i}$ dans les 3 sols: les souches réunies par un trait ne diffèrent pas significativement entre elles pour un niveau de probabilité global de 0,95 .

Strain effect in relation to parameter $b_{i}$ and comparison test of $b_{i}$ in 3 soils : data linked by the same line are not significantly different at 0.95 level.

souches diffèrent significativement du témoin. Deux souches (Fo 47 et Fol 8) se distinguent significativement des autres; Fo 20 semble avoir un comportement intermédiaire.

\section{Sol d'Ouroux}

En sol d'Ouroux, le modèle de l'effet temps est le même que dans le cas précédent (tabl. 2). Le $\chi^{2}$ 
obtenu reste anormalement élevé, cependant les résidus ne présentent aucune tendance en fonction des différentes variables. Le facteur d'hétérogénéité est ici égal à 1,9 , ce qui revient à multiplier les écarts-types par 1,4 . On note que toutes les souches sont améliorantes par rapport au témoin. On obtient 2 groupes de souches avec un léger chevauchement (fig. 3). Ce type de chevauchement est courant dans le cadre des méthodes de comparaison multiple.

\section{Sol de Châteaurenard}

Le modèle obtenu pour le temps est identique à celui des 2 autres sols (tabl. 2). Il n'y a pas non plus de tendance particulière dans les résidus et le facteur d'hétérogénéité est voisin de celui du sol d'Ouroux. Deux souches (Fs 59 et Fs 52) sont nettement inférieures à l'ensemble des autres ; on note un fort chevauchement entre les 2 groupes de tête (fig. 3 ).

\section{B. Analyse de la résistance}

Le paramètre $\mathrm{S}$ désigne dans cette expérimentation l'effet des différents traitements modifiant la résistance du sol. La fonction temps comporte ici un terme de plus, le terme en $\mathrm{T}^{2}$ étant significatif (tabl. 3). Le

\section{TABLEAU 3}

Valeurs des parameites (et écart-type) du modele defini par léquation $\log \frac{P}{l-P}=a+h_{i}+g(D)+h(T)$ pour Vexpérience détude du mécanisme de la résistance du sol de Châteaurenard. L'approximation normale du $\gamma^{2}$ a été calculée par A. $N=、 2 \gamma^{2}-2 d \overline{l l}-1$.

Parameter values (and standard deviation) of the model defined by the equation $\log \frac{P}{l-P}=a+b_{i}+g(D)+h(T)$ in the study of suppression mechanisms in Chateaurenard soil $\chi^{2}$ normal approximation calculated by $N \cdot A=\sqrt{2 \chi^{2}}-\sqrt{2 d d l-1}$

\begin{tabular}{|c|c|}
\hline Paramètres & $\begin{array}{c}\text { Valeur des paramètres } \\
\text { (écart-type) }\end{array}$ \\
\hline $\begin{array}{l}\mathrm{a}_{1}(\text { témoin TRd) } \\
\mathrm{b}_{2}(\mathrm{TRd}+\text { myc. }+ \text { Fo } 47) \\
\mathrm{b}_{3}(\mathrm{TRd}+\text { Fo } 47) \\
\mathrm{b}_{4}(\mathrm{TRd}+\text { myc. }) \\
\mathrm{b}_{5}(\mathrm{TR}) \\
\log (\mathrm{D}) \\
\mathrm{T}^{2} \\
\mathrm{~T}^{3} \\
\mathrm{~T}^{4} \\
\mathrm{e}^{\mathrm{T}}\end{array}$ & $\begin{aligned} 11,14 & (0,72) \\
3,78 & (0,22) \\
2,55 & (0,19) \\
3,06 & (0,20) \\
4,47 & (0,25) \\
-\quad 1,04 & (0,07) \\
-\quad 2,51 & (0,9) \\
1,07 & (0,4) \\
-\quad 0,21 & (0,08) \\
0,36 & (0,13)\end{aligned}$ \\
\hline $\begin{array}{l}\text { ddl } \\
\chi^{2}\left(\chi^{2} \text { modèle minimal }\right) \\
\text { Approximation normale } \\
\text { Facteur d'hétérogénéité }\end{array}$ & $\begin{array}{c}115 \\
182(1642) \\
3,9 \\
1,58\end{array}$ \\
\hline
\end{tabular}

facteur d'hétérogénéité est de 1,58 et on ne détecte pas de tendance dans les résidus. La signification du classement des traitements peut être testée. La figure 4 indique qu'il existe des différences significatives entre le niveau de résistance de la terre désinfectée et les autres traitements; bien qu'à la limite du seuil, les traitements TRd + Fo 47 et TR + myc ne diffèrent pas significativement entre eux.

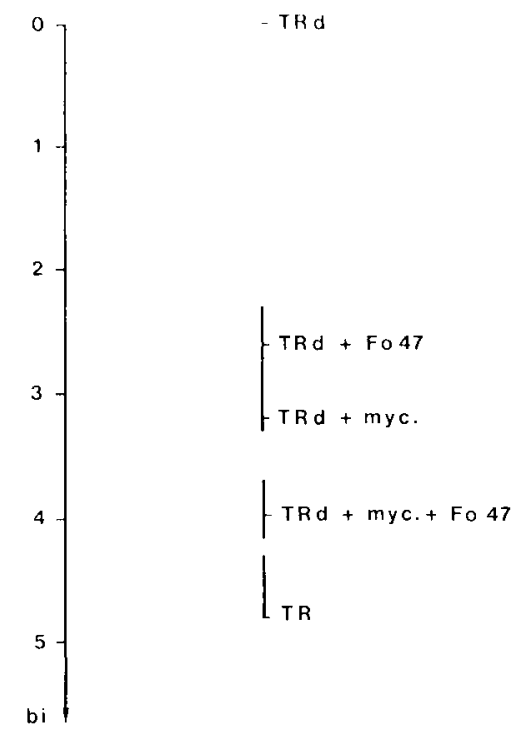

Figure 4

Analyse de l'effet traitement en fonction de la valeur prise par le paramètre $b_{i}$. Les traitements réunis par un trait ne diffèrent pas significativement entre eux pour un niveau de probabilité global de $0,95\left(T R d=\right.$ terre résistante désinfectée à la chaleur : $100{ }^{\circ} \mathrm{C}$, $30 \mathrm{mn} ; \mathrm{TRd}+m y \mathrm{c}=$ terre résistante désinfectée + mycoflore exempte de Fusarium; T.R. = terre résistante)

Treatment effect in relation to parameter $b_{i}$; data linked by the same line are not significantly different at the 0.95 level (TRd = heat treated $100{ }^{\circ} \mathrm{C}, 30 \mathrm{mn}$ suppressive soil ; $T R d+m y c=$ heat treated suppressive soil + complex mycoflora without Fusarium ; $T . R .=$ suppressive soil).

\section{DISCUSSION}

\section{A. Aspects statistiques}

La confrontation des données expérimentales au modèle mathématique défini par l'équation (2) permet de proposer une méthode statistique de comparaison des niveaux de réceptivité des sols aux fusarioses vasculaires.

En outre, cette étude démontre que la probabilité de survie des plantes n'est pas constante au cours du temps. Il s'agit là d'un point important généralement peu discuté dans le cadre des essais biologiques.

Le logiciel GLIM permettant d'analyser les données cumulées rendues indépendantes entre elles par le calcul des probabilités de survie, l'ensemble des données disponibles a été utilisé.

La modélisation de l'effet temps est complexe en raison de sa non-linéarité manifeste. L'évolution de la population pathogène en présence de plantes, après son introduction dans le sol, est actuellement mal connue. Le modèle prend en compte la dose initiale introduite, or il est vraisemblable que la probabilité de survie à un instant donné n'est pas en relation directe avec la densité initiale de l'agent pathogène. De plus, ce modèle est basé sur l'hypothèse d'une absence d'interaction entre l'effet souche et l'effet temps. (Remarquons que GLIM permet de tester l'existence d'interactions entre les facteurs. Sur nos données, la prise en compte d'une éventuelle interaction souche $x$ dose ou souche $\times$ temps n'a pas permis d'améliorer sensiblement l'ajustement du modèle). Dans le cadre 
de cette étude, l'effet-temps a été modélisé dans le but de l'extraire de la variabilité totale afin d'améliorer la précision de l'analyse de l'effet traitement. Malgré cette approche délibérément empirique, la meilleure équation pour l'effet temps est identique dans les 3 sols étudiés, avec un effet significatif des termes en $\mathrm{T}^{3}, \mathrm{~T}^{4}$ et $\exp (\mathrm{T})$. Ces termes donnent une grande souplesse au modèle et permettent d'obtenir des variations assez brusques de la dérivée de $h(T)$.

La variance des données expérimentales s'est révélée être supérieure à sa valeur théorique déduite du schéma binomial ; c'est pourquoi la matrice de covariances a dû être multipliée par un facteur d'hétérogénéité. Cette correction ne peut être effectuée sans précaution, notamment sans une analyse préalable des résidus. Ce facteur d'hétérogénéité d'un niveau faible (compris entre 1,5 et 1,9 ) a des valeurs très proches pour 4 expériences totalement indépendantes; ceci tend à prouver qu'il s'agit bien là d'une variabilité d'ordre uniquement expérimental.

Ainsi, le modèle retenu semble correct. Les résidus ne montrent aucune tendance particulière et les écarts entre valeurs observées et calculées sont sans importance pratique pour ce type d'essai biologique se déroulant sur une longue période. Ce modèle se révèle utile : il permet de classer des niveaux de réceptivité différents par une méthode objective qui vise avant tout à minimiser le risque de $1^{\text {re }}$ espèce.

\section{B. Aspects biologiques}

Le modèle permet de classer les différentes souches de Fusarium non pathogènes pour leur aptitude à protéger les plantes contre les fusarioses vasculaires. Dans les 3 sols étudiés, toutes les souches se sont montrées supérieures au témoin. Ces résultats montrent, par ailleurs, une interaction nette entre sols et souches; ainsi, la souche Fs 59 qui est très proche du témoin désinfecté dans le sol de Dijon, se retrouve en tête dans le sol d'Ouroux. Cela justifie a posteriori l'analyse statistique séparée de l'effet souche dans chacun des sols. Remarquons cependant que dans les 3 sols la souche Fo 47 appartient au groupe de tête.
En ce qui concerne le $2^{\mathrm{e}}$ type d'expérimentation, les résultats enregistrés démontrent la présence complémentaire des mécanismes de résistance générale et de résistance spécifique dans la résistance naturelle du sol de Châteaurenard (ALABOUVETTE, 1986): ainsi l'introduction dans le sol traité à la chaleur d'une souche de $F$. oxysporum non pathogène (TRd + Fo 47) ou d'une mycoflore complexe (TRd + myc) diminue significativement le niveau de réceptivité du sol désinfecté (TRd). Mais l'apport de F. oxysporum non pathogène dans le sol déjà colonisé par la mycoflore complexe (TRd + myc + Fo 47) confère à ce sol un niveau de résistance proche de celui du sol résistant d'origine.

\section{CONCLUSION}

Ce modèle, élaboré à partir de l'expérimentation décrite ci-dessus, constitue maintenant un outil méthodologique utile à l'analyse des mécanismes de réceptivité des sols aux fusarioses vasculaires. Applicable à différents types d'étude, il nous permettra d'analyser statistiquement les différences d'intensité de la compétition nutritive s'exerçant dans le sol pour différents éléments, $\mathrm{Fe}$ et $\mathrm{C}$ principalement (ALABOUVETTE $e t$ al., 1985). En outre, ce modèle mathématique constitue l'élément de base d'une sélection rigoureuse des souches de Fusarium non pathogènes les plus aptes à coloniser le sol et à entrer en compétition avec le Fusarium pathogène au niveau de la plante (COUTEAUDIER et al., 1985). Cette méthode biologique mise au point pour comparer l'efficacité de différentes souches de Fusarium non pathogènes est applicable à l'étude d'autres microorganismes antagonistes, Pseudomonas fluorescents et Trichoderma spp., présentant des caractéristiques et des modes d'action différents. L'intérêt du modèle s'étend donc à la mise au point de tout procédé de lutte microbiologique utilisable dans la lutte contre les fusarioses vasculaires.

Reçu le 9 décembre 1985. Accepté le 24 avril 1986.

\section{RÉFÉRENCES BIBLIOGRAPHIQUES}

Alabouvette C., 1986. Fusarium-wilt suppressive soils from the Châteaurenard région : review of a 10 year study. Agronomie, 6 (3), 273-284.

Alabouvette C., Couteaudier Y., Louvet J., 1982. Comparaison de la réceptivité de différents sols et substrats de culture aux fusarioses vasculaires. Agronomie, 2 (1), 1-6.

Alabouvette C., Couteaudier Y., Lemanceau P., 1985. Nature of intrageneric competition between pathogenic and non-pathogenic Fusarium in a wilt-suppressive soil. In Swinburne T. R. : "Iron, Siderophores and Plant Disease ", NATO ASI SERIES (sous presse).

Baker R. J., Nedler J. A., 1978. The GLIM System (Release 3), Numerical Algorithms Group éd., Oxford, Grande-Bretagne, 137 p.
Baker R., Maurer C. L., Maurer R. A., 1967. Ecology of plant pathogens in soil. VII. Mathematical models and inoculum density. Phytopathology, 57, 662-666.

Couteaudier Y., Letard M., Alabouvette C., Louvet J., 1985. Lutte biologique contre la fusariose vasculaire de la tomate. Résultats en serre de production. Agronomie, 5 (2), 151-156.

Finney D. J., 1978. Statistical Method in Biological Assay. Charles Griffin and Co ed., Londres, $3^{\mathrm{c}}$ ed., $463 \mathrm{p}$.

Tello-Marquina J. C., Alabouvette C., 1984. Observations sur la persistance dans le sol des microconidies de Fusarium oxysporum. Agronomie, 4 (9), 885-890. 